\title{
Research on Changes of Dietary Fiber in Bean Dregs during Extrusion Process
}

\author{
Guang Zhang ${ }^{\mathrm{a}, *}$, Zhihui Sun ${ }^{\mathrm{a}}$, Yanguo Shi ${ }^{\mathrm{a}}$, Mingshou Lu ${ }^{\mathrm{a}}$ \\ School of Food Engineering, Harbin University of Commerce, Harbin, Heilongjiang, China \\ arczkzg@163.com
}

Keywords: Dietary Fiber, Bean Dregs, Extrusion

\begin{abstract}
Bean Dregs contain abundant dietary fibers (DF), proteins, fats, vitamins and microelements, among which DF takes more than 50\% content thus bean dregs are good raw material for extracting DF. DF is named as the seventh major nutrients since it has many important physiological functions. It can promote intestinal peristalsis to reduce the food dwelling time in gastrointestinal tract and speed up excretion. DF has powerful water absorbing swelling nature which can reduce food intake dose. Moreover, xylons in DF can combine with cholic acid to prevent calculus. Since most DF in bean dregs are insoluble and cannot be absorbed by human body, we planned to conduct extrusion process to convert some insoluble DF to soluble DF by a series of unit processes. Research showed that by adjusting heating temperature, screwing speed, water content in bean dregs, a certain portion of insoluble DF changed to soluble DF. This results greatly increased the availability of DF in bean dregs.
\end{abstract}

\section{Introduction}

With the improvements of human living standards, dietary habits began to change since several decades ago. Fine foods have been manufactured with larger amount which provides increasing protein, fat and calories to human daily diet while dietary fibers intake decreased contrarily leading to an imbalance of eating habit inventory and a series of modern illnesses of affluence such as diabetes, cardiovascular diseases, obesity, etc [2]. Massive researches have revealed that dietary fibers have remarkable influence on preventing and treating such diseases. As a result, dietary fiber is named as the putative seventh nutrient. Among various species of food, it has been revealed that soybean dietary fibers possess leading medical function and physiology. Since the annual production of soybean in China is around 12 million tons[3], soybean dregs which were bounded to be wasted of enormous amount during soybean industrial process is an outstanding source for manufacturing dietary fibers in order to improve human health.

Dietary fibers is defined as the constitution of indigestible polysaccharide carbohydrates and lignin. The soluble dietary fibers (SDF) is consisted of colloid compounds and saccharides, while insoluble dietary fibers (IDF) is consisted of lignin, cellulose, hemicellulose, chitosan and protopectin [4,5]. Since SDF has antidiabetics function and can be hydrolyzed in colon to prevent colon cancer, it attracts more attention in food engineering and medical science research fields. Moreover, SDF has better physiology compared with IDF, thus it can be added to food process as functional food additives.

As a by-product of soybean process, soybean dregs is mainly utilized as animal fodder because of its low solubility. However, soybean dregs is a good source of dietary fibers thus the more and more researchers have been focusing on extracting dietary fibers from soybean dregs [8]. For the purpose of extracting different dietary fibers, four classic methods are commonly utilized in the experimental process, which are chemical separation, membrane separation, fermentation method and chemical-enzyme extraction method. Chemical separation and membrane separation can not eliminate all amounts of proteins and fats in the soybean dregs and fermentation method consumes much time, the chemical-enzyme extraction method has been widely opted during dietary fiber extraction process as enzyme hydrolysis can remove residual proteins and fats after chemical separation to obtain purer dietary fibers. 
The SDF extracted from soybean dregs is constituted by non-starch polysaccharide molecules with stable chemical properties. The functions of SDF are depended on its physiology which is closely related to polysaccharide chain formation and inter-chain forces. Compared with the four extracting methods listed above, extrusion is one mechanical degradation method which can integrate homogeneity, smashing, fusion, sterilization and curing unit operation and can realize the transition of macromolecule polymers to SDF directly or indirectly [9]. Meanwhile, the color and flavor of high cellulose can be changed by extrusion. Under the extrusion thermal field, mechanical energy field and high pressure, hydrogen bonds which hold the cellulose fascicular texture are broken leading to granulation of cellulose molecules $[10,11]$. As a result, dietary fiber modification commonly utilizes extrusion method.

In this research, we integrated extrusion method and enzyme extraction method to obtain SDF from soybean dregs. Firstly, soybean dregs was prepared after grinding soybean milk and desiccation; Secondly, all dry soybean dregs samples were processed by extruding machine under different operating situations; Finally, extruded samples were processed by chemical-enzyme extraction method to obtain SDF in soybean dregs.

\section{Methods}

\subsection{Materials and Instruments}

Wet Soybean dregs was prepared by squeezing soybean milk (soybean: water $=1: 10$ ) and samples were filtered by 80-mesh sieve after desiccation. Neutral protease was provided by Solaribio, all the other reagents are analytically pure. Brabender twin screw extruder was selected in this study. Extruder is constituted by combined sleeved ( 5 joints) and screws ( 5 joints). The screw speed is $0 \sim 550 \mathrm{r} / \mathrm{min}$, barrel temperature is $0 \sim 400^{\circ} \mathrm{C}$ and die head diameter is $2 \times 20 \mathrm{~mm}$. Thermostatic water bath oscillator was used of HAD-HZS-H from Beijing Heng Odd Instrument Co., Ltd, centrifuge was used of TDL-5_A from Shanghai Anting Scientific Instrument Factory.

\subsection{Experiments}

Single factor experiments were carried out with three factors which are water content, extruding temperature and screw rotation speed. Firstly, dry soybean dregs was prepared with different water content $(20 \%, 22 \%, 24 \%, 26 \%, 28 \%$ and 30\% w/w) before being extruded. The influence of water content was examined under the extruding temperature of $110^{\circ} \mathrm{C}$ and screw rotation speed of 130 $\mathrm{r} / \mathrm{min}$. Then, extruding temperature was examined with the range of $90^{\circ} \mathrm{C}$ to $130^{\circ} \mathrm{C}$ with $10^{\circ} \mathrm{C}$ interval with the settled water content of $26 \%$ and screw rotation speed of $130 \mathrm{r} / \mathrm{min}$. Finally, screw rotation speed was examined from $110 \mathrm{r} / \mathrm{min}$ to $150 \mathrm{r} / \mathrm{min}$ with the fixed water content of $26 \%$ and extruding temperature of $110^{\circ} \mathrm{C}$.

In the next step, 16 soybean dregs samples were gathered and $3 g$ of each sample was taken to conduct alkali treatment. Distilled water was added to each sample with the amount of $45 \mathrm{~g} .1 \mathrm{~mol} / \mathrm{L}$ $\mathrm{NaOH}$ was diluted to each sample till the $\mathrm{pH}$ reached 13 and all samples were put into $80^{\circ} \mathrm{C}$ water bath of $80 \mathrm{~min}$. Samples were then washed by distilled water after water bath and the precipitates were IDF abstracted from soybean dregs. $50 \mathrm{~mL}$ distilled water was diluted to each IDF sample and $0.5 \%$ neutral protease was added to samples. Enzyme hydrolysis process was conducted under $40^{\circ} \mathrm{C}$ for 2 hours. The supernatants of the samples were gathered after centrifugation the 4 times (v/v) absolute ethyl alcohol was added to the supernatants for alcohol precipitation process. Consequently, SDF samples were gathered after centrifugation and measured of the weights.

\section{Results and Discussions}

After extruding soybean dregs, the degree of polymerization, molecular weight of fiber reinforced polymers will change leading to an increase of SDF yield from soybean dregs [1]. It has been published that the SDF yield in soybean dregs after extrusion was greatly improved compared with raw soybean dregs under same alkali treatment condition. Moreover, extrusion process could also passivate some lipoxidase which brings about beany flavors. In this study, the yield of SDF was 
calculated as equation 1.

$$
\text { SDF Yield }=\frac{\text { SDF weight }}{\text { soybean dreg sample weight }} \times 100 \%
$$

Three factors related to extrusion process were observed in this study. Five extruding temperatures were determined from $90^{\circ} \mathrm{C}$ to $130^{\circ} \mathrm{C}$ while rotation speed was fixed at $130 \mathrm{r} / \mathrm{min}$ and water content was fixed at $26 \%$. Five rotation speeds were determined from 110 to $150 \mathrm{r} / \mathrm{min}$ while temperature was fixed at $110^{\circ} \mathrm{C}$ and water content was fixed at $26 \%$. Also water contents were determined from $20 \%$ to $30 \%$ while temperature was fixed at $110^{\circ} \mathrm{C}$ and rotation speed was fixed at $130 \mathrm{r} / \mathrm{min}$. The factor ranges were determined according to the extruder (Brabender) properties and former experiments.

Temperature is one of the key influencing factors for extrusion process, as figure 1 illustrated, extruding temperature was investigated and the influence on SDF yield was shown. The yield was at the lowest level under $90^{\circ} \mathrm{C}$ and increased to $31.27 \%$ under $110^{\circ} \mathrm{C}$. When temperature exceeded $110^{\circ} \mathrm{C}$, the yield of SDF started to decrease until the $130^{\circ} \mathrm{C}$ point with $8.61 \%$. As a result, high temperature will lead to the decrease of the SDF yield since it would introduce denaturation of gelatin, pectin and hemicellulose. Temperature of the materials in the extruding socket increased rapidly together with the shear force and compression, glucosidic bonds of the fiber polymers break up and are degraded to carbohydrates of low molecular weights. With the increase of temperature, chemical bonds ruptures also increase leading to more molecular degradations and exposes of water-based groups, being expressed as the increase amount of SDF yields. However, higher temperature generated a darker color of the soybean dregs due to the Mailard reaction leading to a sharp decrease of the SDF yield.

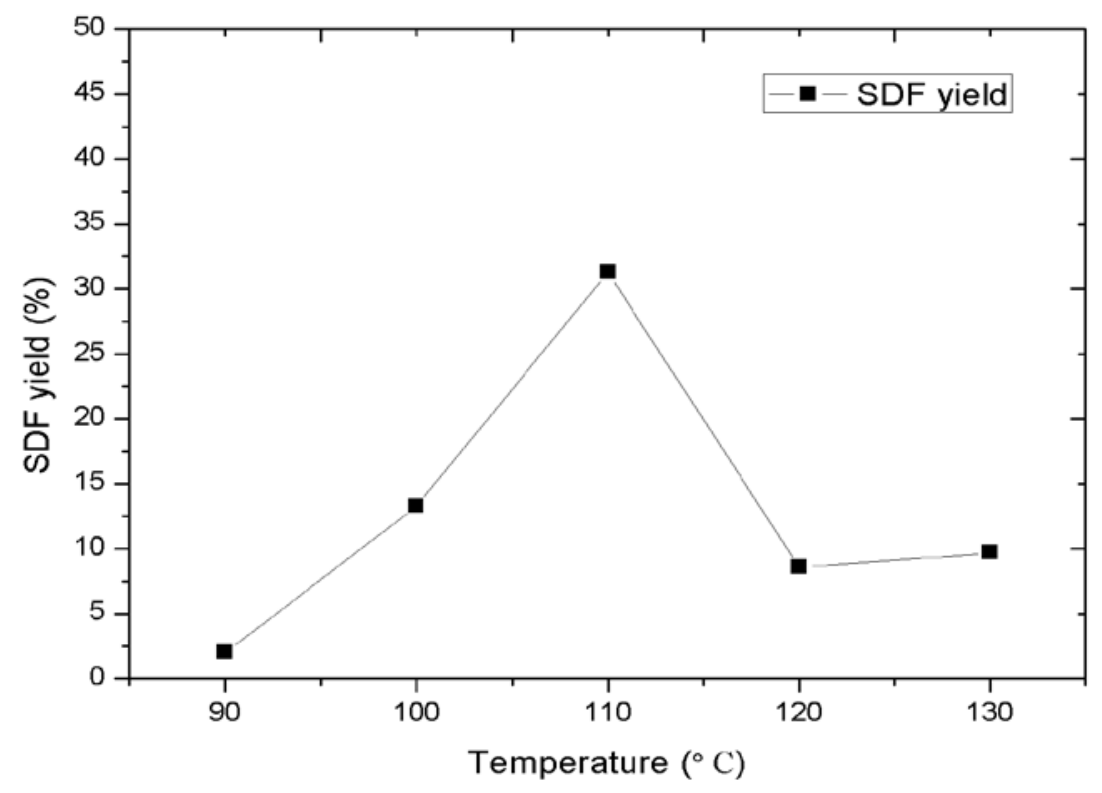

Fig.1. Temperature influence of extrusion on SDF yield from soybean dregs.

Being influenced by viscosity and friction, high mechanical energy is consumed when extruding and cutting high polymers such as protein and starch. Water, as the flexibilizer of polymers, can reduce the interaction forces of materials with lower mechanical energy. In this study, under $110^{\circ} \mathrm{C}$ and $130 \mathrm{r} / \mathrm{min}$, water content was observed to reveal the influence on SDF yield. Figure 2 shows the experimental data with water content from $20 \%$ to $30 \%(\mathrm{w} / \mathrm{w})$. Firstly, the SDF yield increased from $20 \%$ to $24 \%$ with the peak value of $25.95 \%$, and then decreased until $30 \%$ water content with SDF yield of $13.63 \%$. Firstly, the water content was not sufficient to provide flexibilization effect to abstract SDF. However, with the increase of water content, the viscosity of soybean dregs in 
extruding sleeves and screws became lower. Consequently, pressure of grinding head of extruder decreased leading to a negative influence of dietary fiber degradation.

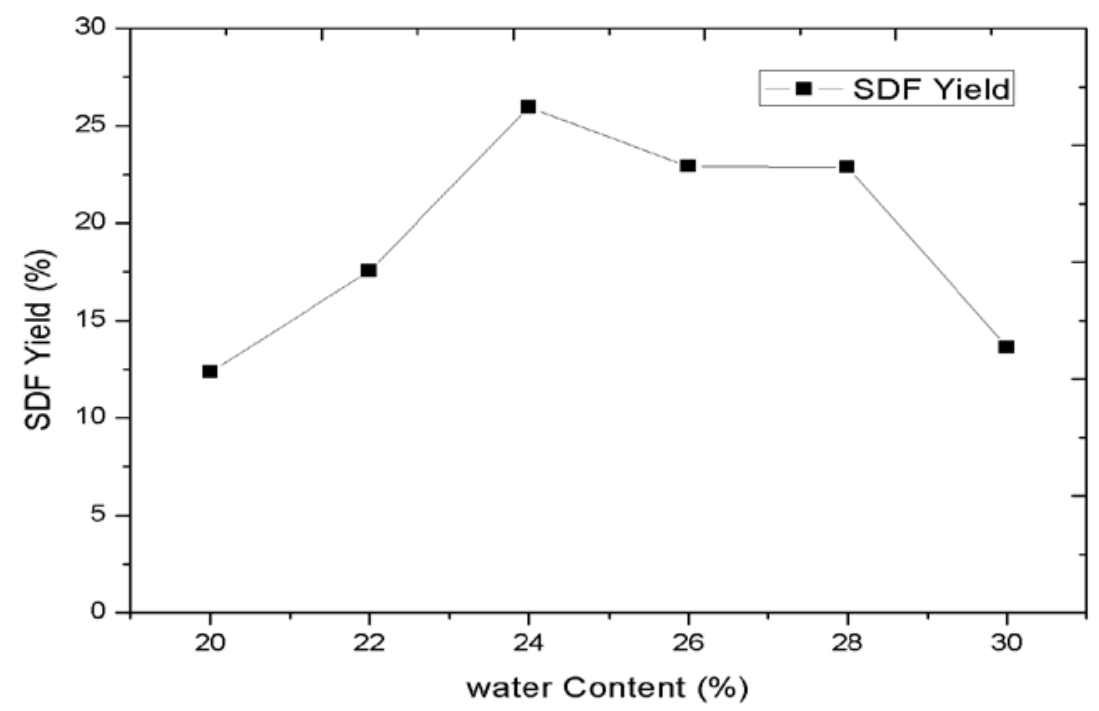

Fig.2. Water content influence of extrusion on SDF yield from soybean dregs.

In addition, screw rotation speed is another essential factor for abstracting SDF from soybean dregs. The expanding rate of soybean dregs can be affected by screw rotation speed. When the rotation speed is accelerated, the exit pressure increases simultaneously. As a result, the unit volume of material discharge also increases in unit time. The expansion decreases inside the screw because of the increasing shear force, thus the SDF yield had the highest value at $110 \mathrm{r} / \mathrm{min}$ and decreased until $150 \mathrm{r} / \mathrm{min}$. For the reason that the optimum rotation speed of the extruder in the research lab is from $110 \mathrm{r} / \mathrm{min}$, the low rotation speed range was not included in this study. The result is illustrated by Figure 3 .

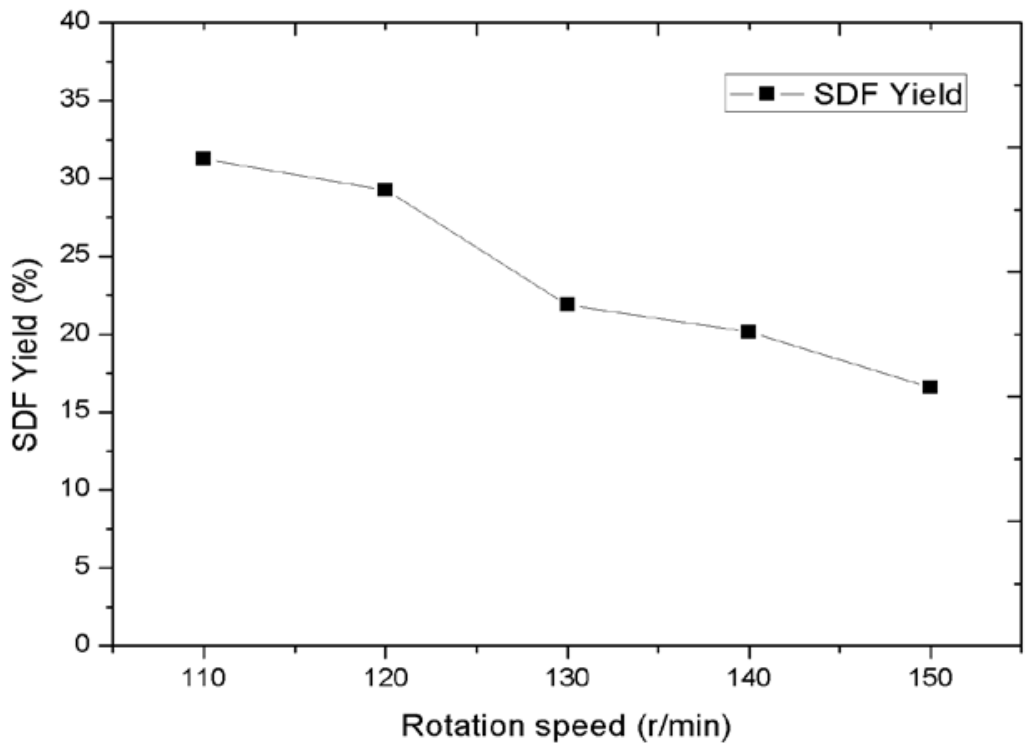

Fig.3. Rotation speed influence of extrusion on SDF yield from soybean dregs.

\section{Conclusion}

In this study, the extruding factors were observed to reveal the influence of SDF yield from 
soybean dregs. The experimental results showed that best extruding temperature was $100^{\circ} \mathrm{C}$, the optimal water content and screw rotation speed were $24 \%$ and $110 \mathrm{r} / \mathrm{min}$ respectively. After extruding, the samples were hydrolyzed by protease under optimal operating situations. Results illustrated that the optimum values of studied factors were reasonable and the results are helpful for future soybean SDF study.

\section{References}

[1] Lou Haiwei and Chi Yujie. Effects of Extrusion-Cooking on Soluble Dietary Fiber in Soybean Residue. Journal of the Chinese Cereals and Oils Association. 24(6), 2009.

[2] Zhang Lan, Jin Si-wen, Yu Han-song, Liu Jun-mei, Piao Chun-hong, Wang Yu-hua, Hu Yao-hui. Effect of High-moisture Extrusion on Dietary Fiber in Textured Protein Made from Soybean Residues. Food Research and Development. 36(24), 2015.

[3] Jiang Zhumao, Chen Xinmei and Miao Jing. Producing Soluble Dietary Fibre from Bean Dregs. Journal of Chinese Cereals and Oils Association. 16(3), 2001.

[4] Tu Zongcai, Duan Dengle, Wang Hui, Chen Lili and Huang Xiaoqin, Structural Characterization and Antioxidant Activity of Soybean Dregs Dietary Fiber. Journal of Chinese Cereals and Oils Association. 30(6), 2015.

[5] Fang Yanqiang, Yang Haiying, Xie Tian, Ren Chengang, Zuo Naibei and Ding Qingbo. Research Progress on Modifications of Dietary Fiber in Extrusion Cooking. Journal of the Chinese Cereals and Oils Association. 31(2), 2016.

[6] Wang Bo, Zhang Ying, Xiao Yan-lin, Xu De-qiong, Chen Gong, You Jing-gang, The Preparation of Dietary Fiber from Soybean Dregs. Food and Fermentation Technology, 49(3), 2013.

[7] Li Na, Ning Zhengxiang, Zhu Ziping and Li Lin. Preparation and Characterization of Dietary Fibers from Soybean Dregs. Food Science.30 (20), 2009.

[8] Zhang Shi-xian, Yang Chun-mei, Wu Jin-hong and Wang Zheng-wu. Advance in Extraction Method and Function of Dietary Fiber from Soybean Dregs. Journal of Southwest China Normal University (Natural Science Edition). 34(4), 2009.

[9] Liu Hao-fei. Characteristics of Soybean Soluble Dietary Fiber. Soybean Science. 34(1), 2015.

[10] Ren Yuan-yuan, Chen Xue-wu and Li Dan-dan. Study on the Extraction of Soybean Soluble Dietary Fiber from Bean Dreg. China Food Additives. 1,2015.

[11] Liu Nan, Sun Yon, Li Yue-xin and Wu pei-feng. Properties, Physiological Function and Application of Dietary Fiber. Journal of Food Safety and Quality. 6(10), 2015. 